\title{
Raman Scattering Investigation and Symmetry Analysis of Ferroelectric/Ferroelastic $\mathrm{Sb}_{5} \mathrm{O}_{7} \mathrm{I}$ Polytype $2 \mathrm{MA}$
}

\author{
W. Prettl \\ Institut für Angewandte Physik der Universität Regensburg, W.-Germany \\ K.H. Rieder \\ IBM Zurich Research Laboratory, Rüschlikon, Switzerland \\ R.N. Nitsche \\ Kristallographisches Institut der Universität Freiburg, W.-Germany \\ Received March 28, Revised Version August 14, 1978
}

The polytype $2 \mathrm{MA}\left(\beta-\mathrm{Sb}_{5} \mathrm{O}_{7} \mathrm{I}\right)$ has the simplest acentric structure of the antimony oxideiodide family. It undergoes an antiferrodistortive phase transition at $438 \mathrm{~K}$ and is both ferroelectric and ferroelastic below that temperature. The complete polarized Raman spectra in the ferroic phase have been measured and compared with those of the ferroelastic, centric polytype $2 \mathrm{MC}\left(\alpha-\mathrm{Sb}_{5} \mathrm{O}_{7} \mathrm{I}\right)$. Several lines could be attributed to $\mathrm{Sb}-0$ and $\mathrm{Sb}-\mathrm{I}$ vibrations. A factor group analysis has been performed and compatibility relations have been established connecting phonon species in the low and high temperature phase. As a function of temperature the spectra revealed a strongly temperature dependent central line and several phonon lines whose intensities vanish above $T_{c}$. Using these phonon line intensities the temperature variation of the order parameter could be determined. The experimental results indicate that the phase transition is of first order.

\section{Introduction}

Penta-antimony-hepta-oxide-iodide, $\mathrm{Sb}_{5} \mathrm{O}_{7} \mathrm{I}$ represents in many respects a unique ferroic material. It exhibits polytypism and up to now eight different polytypic modifications are known. Their crystal structures differ in the number and the stacking of typical, two-dimensional slabs of antimony and oxygen atoms [1-3]. The most interesting property of the $\mathrm{Sb}_{5} \mathrm{O}_{7} \mathrm{I}$ crystal family is the fact that all known polytypes undergo structural phase transitions with transition temperatures $T_{c}$ ranging between $438 \mathrm{~K}$ and $481 \mathrm{~K}$ and they all are ferroelastic in the low temperature phase. Depending on the stacking sequence and the orientation of successive slabs, centric and acentric polytypes ${ }^{\star}$ result. The centric crystals

\footnotetext{
* For a structurally relevant identification of the polytypes (originally $\alpha, \beta, \gamma \ldots$, a modified Ramsdell notation has been introduced [3], in which the symbol sequence denotes: number of slabs per cell, symmetry (e.g. $M=$ monoclinic) and $C$ or $A$ for centric or acentric, respectively.
}

are all purely ferroelastic in the sense of Aizu's definition [4]. The acentric crystals are both ferroelastic and ferroelectric.

In previous publications we reported on Raman scattering measurements and the development of a phenomenological theory to account for the phase transitions of the purely ferroelastic, centric two-slab polytype $2 \mathrm{MC}$, which was initially called $\alpha-\mathrm{Sb}_{5} \mathrm{O}_{7} \mathrm{I}$ $[5,6]$. This crystal, the structure of which is the basis of the centric polytypes, is found to be most abundant in the crystal growing process. The present paper deals with the acentric, two-slab polytype $2 \mathrm{MA}$ which was initially called $\beta-\mathrm{Sb}_{5} \mathrm{O}_{7} \mathrm{I}$. It has the simplest acentric structure and may be considered as the structural basis of the acentric polytypes.

The complete Raman scattering spectra of the ferroic phase have been measured. They are compared with the Raman spectra of the polytype $2 \mathrm{MC}\left(\alpha-\mathrm{Sb}_{5} \mathrm{O}_{7} \mathrm{I}\right)$ and infrared absorption bands of $\mathrm{Sb}_{2} \mathrm{O}_{3}$ and $\mathrm{SbI}_{3}$. By 


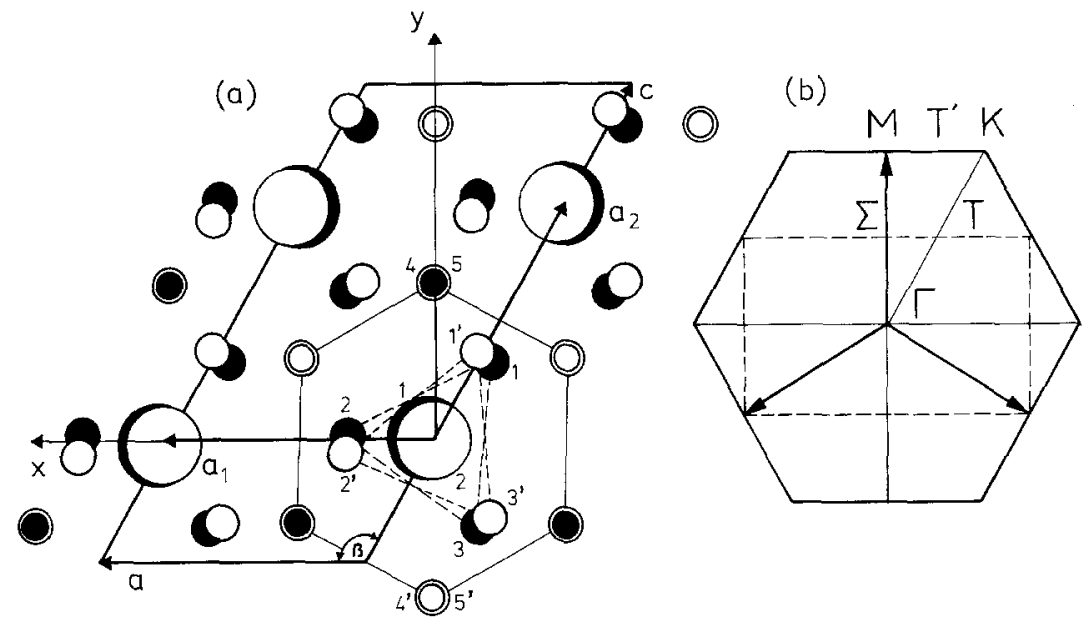

Fig. 1. a Projection of the Sb-(small circles) and I-(large circles) atoms on the monoclinic plane. For the sake of clarity $\mathrm{O}-$ atoms are omitted. Approximate heights $z$ of the atoms given in units of $b$ normal to the plane of the drawing: I1:0.0; I2:0.5; Sb 1-3: 0.25; Sn 1'-3': 0.75; Sb4: 0.05; Sb5: $0.45 ; \mathrm{Sb}^{\prime}: 0.55 ; \mathrm{Sb} 5^{\prime}: 0.95$. (After Nitsche et al. [3])

b Brillouin zone. Points of specific symmetry of the hexagonal structure are denoted by the usual symbols. The broken line displays the zone resulting from the cell doubling a factor group analysis the number and symmetry of zero-wavevector phonons is enumerated for both phases. The temperature dependence of the spectra up to above the transition temperature is shown and the origin of several phonon lines, whose intensities vanish above $T_{c}$, is discussed by applying compatibility relations among irreducible representations of the ferroic and the prototypic structure. A short preliminary account of these results has been reported previously [7].

\section{Crystal Structure and Ferroic Properties}

The crystal structure and the space groups of the polytype $2 \mathrm{MA}\left(\beta-\mathrm{Sb}_{5} \mathrm{O}_{7} \mathrm{I}\right)$ have been determined by Krämer [3] for both phases using $\mathrm{x}$-ray diffraction. In this section we summarize the ferroic properties of its structure as far as it is necessary to discuss the Raman spectra and to understand the mechanism of the phase transition.

In the high temperature prototypic phase the polytype $2 \mathrm{MA}$ belongs to the space group $C_{3 h}^{1}(P \overline{6})$ of the hexagonal system. At $T_{c}=438 \mathrm{~K}$ the crystal undergoes an antiferrodistortive displacive phase transition which doubles one of the primitive translations normal to the hexagonal axis and reduces the space group symmetry to $C_{s}^{2}(P c)$ of the monoclinic system. The crystal lacks inversion symmetry and is piezoelectric in both phases. This has been confirmed independently from the structural investigation by observing a second harmonic signal below and above $T_{c}$ using a $Q$-switched Nd:YAG laser. The nonlinear susceptibilities are of the order of those of quartz [8].

Figure 1 a shows a projection of the crystal lattice on the monoclinic a-c or hexagonal plane, respectively. For the sake of clarity only Sb- and I-atoms are shown. a, $\mathbf{c}$ are monoclinic, $\mathbf{a}_{1}, \mathbf{a}_{2}$ hexagonal axes and $x, y$ are Cartesian coordinates. The monoclinic $\mathbf{b}$-axis, the hexagonal $\mathbf{a}_{3}$-axis and the $z$-coordinate are normal to the plane of the drawing. The primitive periodicity along the crystallographic $\mathbf{b}\left(\mathbf{a}_{3}\right)$ direction comprises two slabs as in the case of the polytype $2 \mathrm{MC}$. The boundary between the slabs is at $z=0.5$. The lower slab is identical so that of the $2 \mathrm{MC}$ modification. In contrast to the polytype $2 \mathrm{MC}$ the upper slab is a mirror-image of that of the $2 \mathrm{MC}$ modification, reflected at a plane normal to the hexagonal $\mathbf{a}_{1}$-axis and containing the hexagonal $\mathbf{a}_{3}$-axis. This yields an acentric structure with an electric dipole moment approximately parallel to the monoclinic a-axis. In higher order polytypes the primitive period along $\mathbf{a}_{3}$ comprises multiples of two slabs. The 2MA-polytype is an improper ferroelectric-ferroelastic crystal $[9,10]$ and belongs according to Aizu's classification of macroscopic ferroic properties [11] to the species $\overline{6} \mathrm{Fm}$ having three orientation states or domains which differ in electric polarization and elastic strain. The polar axis of the ferroic structure is normal to the hexagonal axis of the prototypic phase, but it is not uniquely defined by symmetry within the monoclinic plane. The crystal structure data [3] and pyroelectric measurements [12] show, however, that the spontaneous dipole moment must be oriented very closely parallel to the monoclinic a-axis. Spontaneous polarization and spontaneous strain are completely coupled. In the ferroic phase the crystal may be reversibly switched from one orientation state to another by applying either an electric field or an uniaxial mechanical stress of a suitably chosen orientation. The prototypic point group $C_{3 h}$ may be decomposed into the direct product $C_{3 h}=C_{3} \times C_{s}$. This shows that the threefold rotations around the hexagonal axis transform one domain into another as in the case of $2 \mathrm{MC}-\mathrm{Sb}_{5} \mathrm{O}_{7} \mathrm{I}$. Thus the spontaneous polarization is not reversible but only reorientable by 
$\pm 120^{\circ}$. This is in contrast to other improper ferroelectric-ferroelastic materials e.g. $\mathrm{Gd}_{2}\left(\mathrm{MoO}_{4}\right)_{3}$ whose electric polarization is reversible $[9,10]$.

The mechanism of the phase transition is completely analogous to that of the polytype $2 \mathrm{MC}$ and seems to be a general feature of all polytypes. In the prototypic high temperature phase the elementary cell contains two formula units $\mathrm{Sb}_{5} \mathrm{O}_{7} \mathrm{I}$ with the two I-atoms located along the hexagonal axis. This cell is outlined in Fig. 1 a by the hexagon. Below $T_{c}$ two equivalent Iatoms in neighbouring unit cells are translated alternately in opposite directions normal to the hexagonal axis. These iodine displacements in conjunction with displacements of the oxygen sublattice (not shown) result in a doubling of the unit cell and yield the monoclinic low temperature structure. Iodine displacements may take place along the three twofold axes in the hexagonal plane, $\mathbf{a}_{1}$ (as shown in Fig. 1a) $\frac{\mathbf{a}_{1}+\mathbf{a}_{2}}{2}$ or $\mathbf{a}_{2}$ and generate the three macroscopically distinguishable domains of the ferroic phase.

Applying an external uniaxial stress perpendicular to one of these axes results in a reorientation of the crystal. The I-atoms are forced to occupy equilibrium positions parallel to the chosen axis and normal to the stress. Likewise an electric field along one of the twofold axes orients the I-atoms parallel to the field. The doubling of the elementary cell causes the Brillouin zone to be folded normal to the $\Sigma$-axis as shown in Fig. $1 \mathrm{~b}$. The zone boundary $M$-point is translated to the zone center. Therefore the order parameter of the phase transition is expected to transform according to a space group representation belonging to the $M$-point in the Brillouin-zone of the prototypic phase. As in the case of the $2 \mathrm{MC}$ modification, the order parameter couples quadratically to the elastic deformation tensor and this produces a ferroelastic low temperature structure. In addition, due to the lack of inversion-symmetry of the hexagonal phase, in the 2MA-polytype also a coupling to the electric polarization is allowed by symmetry, yielding the ferroelectric properties of these crystals.

\section{Experimental Techniques}

The experiments were performed by the usual $90^{\circ}$ Raman scattering geometry. An argon laser as an exciting source at $514.5 \mathrm{~nm}$ and a Jarrel-Ash double monochromator to analyze the scattered radiation were used. The laser power was as low as 50 to $100 \mathrm{~mW}$ in order to avoid heating of the sample. The optical resolution was $0.6 \mathrm{~cm}^{-1}$. The polarization direction of the incoming laser beam was selected by a broadband polarization rotator and that of the scattered radiation by a Polaroid filter. Standard photon counting techniques were applied.

The as-grown crystals are prisms and pyramids with (pseudo-)hexagonal cross sections. They are optically transparent up to $390 \mathrm{~nm}$ at $300 \mathrm{~K}$ where strong absorption due to the electronic absorption edge sets in. The energy of the absorption edge decreases linearly with rising temperature and corresponds to $430 \mathrm{~nm}$ at $T=570 \mathrm{~K}$ [12], which is far away from the wavelength of the exciting laser. Therefore a change of intensity in the spectra as a function of temperature resulting from resonant effects is not to be expected. Samples of approximately $2 \mathrm{~mm}$ length of each side were prepared with edges parallel to the Cartesian coordinates of Fig. 1a. The crystals may easily be cleaved parallel to the monoclinic (or hexagonal) plane, i.e. parallel to the slabs which form the crystal structure. Cleaved faces could be used without further preparation. Smooth and optically clear faces normal to the cleavage plane were prepared by carefully grinding and polishing the crystals using very low pressure, because the crystals cracked very easily along domain boundaries. The samples were finally transformed into single domain crystals by applying a small uniaxial stress normal to the a-axis (along the $y$-coordinate, Fig. 1a). The orientation of the samples can immediately be determined by observing the extinction angles between crossed polarizers. The main vibrational directions of the optically biaxial crystal in the monoclinic phase are along the a-axis, the b-axis [3] and the axis perpendicular to these two axes and, thus, coincide for the domain shown in Fig. $1 \mathrm{a}$ with the Cartesian coordinate system. However, switching from one domain to another cannot be performed so easily as in the polytype $2 \mathrm{MC}$. It was not possible to obtain a single domain crystal, since at the edges of a sample always misoriented parts remained.

The Raman-measurements were performed at room temperature and at higher temperatures up to above $T_{c}$. The sample was placed in an electrically heated oven with windows of fused quartz. The temperature was monitored by a $\mathrm{NiCr}-\mathrm{Ni}$ thermocouple and controlled by a Thor model 3010 temperature controller. A temperature stability of approximately 0.5 degrees has been achieved.

\section{Raman Scattering in the Ferroic Phase}

\section{a) Selection Rules}

The Raman selection rules are determined by the Raman polarizability tensor R. Usually the ionic contribution $\mathbf{R}^{i}$ is tabulated for the various crystal 


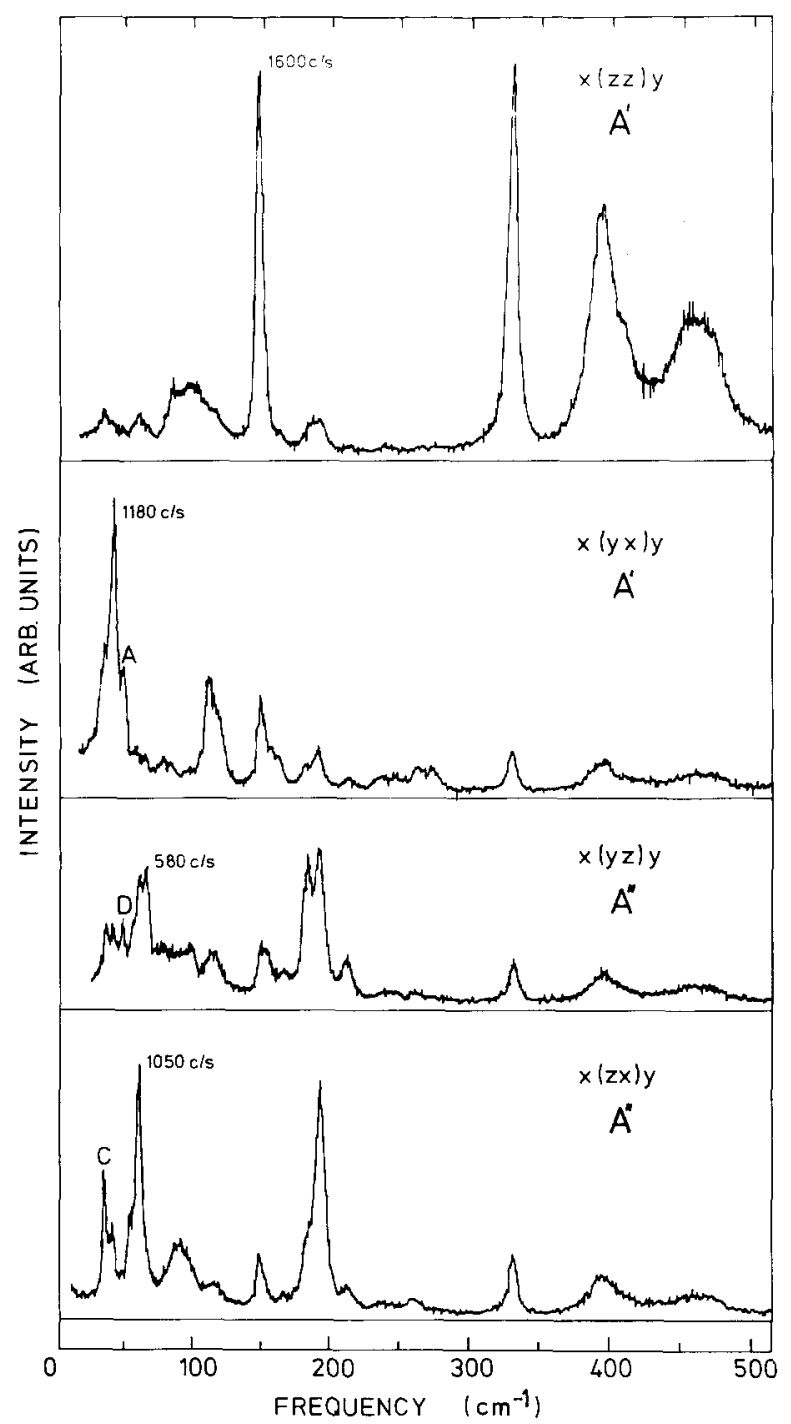

Fig. 2. Raman spectra of $\mathrm{Sb}_{5} \mathrm{O}_{7} \mathrm{I}$ polytype $2 \mathrm{MA}$ in the ferroic phase at room temperature. The numbers give the count rates for selected lines. The letters indicate lines, whose temperature dependence is shown in Figs. 3 and 4

classes [13]. For the ferroic point group $C_{s}$ of $2 \mathrm{MA}$ $-\mathrm{Sb}_{5} \mathrm{O}_{7} \mathrm{I} \mathbf{R}^{i}$ has the form:

$\mathbf{R}^{i}\left(A^{\prime}\right)=\left(\begin{array}{lll}a & d & 0 \\ d & b & 0 \\ 0 & 0 & c\end{array}\right), \quad \mathbf{R}^{i}\left(A^{\prime \prime}\right)=\left(\begin{array}{lll}0 & 0 & e \\ 0 & 0 & f \\ e & f & 0\end{array}\right)$

where the coordinates of Fig. $1 \mathrm{a}$ are used and $A^{\prime}$ and $A^{\prime \prime}$ denote the two irreducible representations of $C_{s}$. However, due to the lack of a center of symmetry all optical modes of the polytype $2 \mathrm{MA}$ are polar phonons and are both Raman and infrared active. The macroscopic electric field associated with longitudinal polar modes causes an electrooptic contribution $\mathbf{R}^{e}$ to the Raman tensor which must also be taken into account. Shapiro and Axe [14] have given a very useful expression to determine $\mathbf{R}^{e}$ in the limit $q \gg \omega / c$, a condition which is usually fulfilled in right-angle scattering.

In contrast to $\mathbf{R}^{i}, \mathbf{R}^{e}$ depends on the phonon wavevector $\mathbf{q}$ and, for a given $\mathbf{q}$, its form is solely determined by the crystal class independent of the particular symmetry species. In general, the form of $\mathbf{R}^{e}$ differs from that of $\mathbf{R}^{i}$; thus in order to separate the spectra of phonons belonging to different symmetry species, not only the polarization of the incident and scattered radiation has to be considered but also the momentum transfer $\mathbf{q}$ must be chosen suitably. In the ferroic phase of the polytype $2 \mathrm{MA}, A^{\prime}$ and $A^{\prime \prime}$ spectra may be separated with scattering configurations $x\left(x_{i} x_{j}\right) y$, with $x_{i}=y, z$ and $x_{j}=x, z$. In this case $\mathbf{q}$ lies in the monoclinic plane and $A^{\prime \prime}$ modes are purely transversal, thus giving $\mathbf{R}^{e}\left(A^{\prime \prime}\right)=0$. Furthermore, it may easily be proved that $\mathbf{R}^{e}\left(A^{\prime}\right)$ has just the form of $\mathbf{R}^{i}\left(A^{\prime}\right)$ and therefore $A^{\prime}$ and $A^{\prime \prime}$ modes may be excited independently. If the incident beam is along the monoclinic b-axis and the $90^{\circ}$ scattered beam lies in the monoclinic plane, $A^{\prime}$ and $A^{\prime \prime}$ spectra should be mixed due to $\mathbf{R}^{e}$. This happens for the configuration $z\left(x_{i} x_{j}\right) y$ with $x_{i}=x, y$ and $x_{j}=x, z$.

\section{b) Raman Spectra}

In Fig. 2 the Raman spectra at room temperature of $x\left(x_{i} x_{j}\right) y$ configurations are shown in the frequency range from 10 to $520 \mathrm{~cm}^{-1}$. To make the intensities of the records comparable the count rate of the peak intensity of one line is indicated in each spectrum.

Raman modes belonging to $A^{\prime}$ and $A^{\prime \prime}$ species may clearly be distinguished in the spectra and we are able to assign all well resolved lines. Strong lines in the $A^{\prime}$-spectra weakly spill over in $A^{\prime \prime}$ records, where they should be forbidden, and vice versa. See for instance the $A^{\prime}$ line at $146 \mathrm{~cm}^{-1}$, or the $A^{\prime \prime}$ doublet at $182 \mathrm{~cm}^{-1}$ and $190 \mathrm{~cm}^{-1}$. We think that this spilling over is due to a probably not complete single domain scattering volume and a slight misorientation of the sample.

The $z\left(x_{i} x_{j}\right) y$ spectra, where $\mathbf{R}^{e}$ is expected to be effective, have also been measured. However, the mixing of the spectra was approximately of the same magnitude as that of Fig. 2, indicating that the influence of $\mathbf{R}^{e}$ on the scattering spectra cannot be determined within the accuracy of our measurements. The low frequency part of the $z\left(x_{i} x_{j}\right) y$ spectra is shown in Figs. 3 and 4. The complete spectra are not displayed, because they do not yield new lines in addition to those of Fig. 2.

At higher frequencies the Raman spectra of $2 \mathrm{MA}$ $-\mathrm{Sb}_{5} \mathrm{O}_{7} \mathrm{I}$ show very similar features to those of $2 \mathrm{MC}$ 
Table 1. Frequencies of Raman scattering lines of monoclinic $2 \mathrm{MA}$ - and $2 \mathrm{MC}-\mathrm{Sb}_{5} \mathrm{O}_{7} \mathrm{I}$ at room temperature and frequencies of Raman lines and infrared (ir) absorption bands of $\mathrm{Sb}_{2} \mathrm{O}_{3}$ after [1315]. Frequencies of strong lines which yield similar structures in the Raman spectra of both polytypes are underlined

\begin{tabular}{|c|c|c|c|c|c|}
\hline \multicolumn{4}{|c|}{$\mathrm{Sb}_{5} \mathrm{O}_{7} \mathrm{I}$} & \multicolumn{2}{|l|}{$\mathrm{Sb}_{2} \mathrm{O}_{3}$} \\
\hline $\begin{array}{l}2 \mathrm{MA} \\
A^{\prime} \\
\end{array}$ & $\begin{array}{l}2 \mathrm{MC} \\
A_{\mathrm{g}} \\
\end{array}$ & $\begin{array}{l}2 \mathrm{MA} \\
A^{\prime \prime} \\
\end{array}$ & $\begin{array}{l}2 \mathrm{MC} \\
B_{g} \\
\end{array}$ & Raman & i.r. \\
\hline 34.5 & 31 & 33 & 31 & & \\
\hline 37 & 39.5 & 38.5 & & & \\
\hline \multirow[t]{2}{*}{41.5} & 41 & & 40 & & \\
\hline & & 46 & 45.5 & & \\
\hline \multirow[t]{4}{*}{48} & & & 48.5 & & \\
\hline & & 53 & & & \\
\hline & & 58 & 57 & & \\
\hline & & 63 & 70 & & \\
\hline 78 & 79 & 75 & 80 & & \\
\hline \multirow[t]{2}{*}{86} & & 88 & & & \\
\hline & 104 & & & & \\
\hline \multirow[t]{2}{*}{111} & 110 & & 111 & & \\
\hline & & & & 127 & \\
\hline \multirow{2}{*}{$\frac{146}{149}$} & $\underline{145}$ & $\underline{147}$ & 145 & 145 & \\
\hline & & 152 & & & \\
\hline \multirow{4}{*}{$\frac{156}{161.5}$} & $\underline{156}$ & & & & \\
\hline & & 165 & & & \\
\hline & & 182 & 182.5 & 180 & 181 \\
\hline & & $\overline{190}$ & $\overline{190}$ & 197 & \\
\hline \multirow[t]{2}{*}{213} & 210 & $\overline{210}$ & $\overline{212}$ & & \\
\hline & & 232 & & 242 & \\
\hline 262 & & & 259 & & 265 \\
\hline \multirow[t]{2}{*}{273.5} & 272 & & & 271 & \\
\hline & & & & & 305 \\
\hline \multirow[t]{2}{*}{330.5} & 330 & & & & 340 \\
\hline & & & & 365 & 353 \\
\hline 394 & 387 & & & & 388 \\
\hline 408 & 415 & & & 419 & \\
\hline \multirow{7}{*}{459} & $\{\overline{456}$ & & & & 480 \\
\hline & $\frac{\overline{478}}{534}$ & & & & \\
\hline & 534 & & 568 & & 565 \\
\hline & 600 & & & & 597 \\
\hline & & & & 688 & 695 \\
\hline & 750 & & & 741 & 750 \\
\hline & & & & & 835 \\
\hline
\end{tabular}

$-\mathrm{Sb}_{5} \mathrm{O}_{7} \mathrm{I}$, as it is expected from the structural relationships of both crystals. The group of lines above $330 \mathrm{~cm}^{-1}$ is very striking. It is separated from the remaining lines by a gap of about $100 \mathrm{~cm}^{-1}$ in which only very weak structures are observed. Above 330 $\mathrm{cm}^{-1}$ the structure of the scattering intensity as a function of frequency is almost the same as in the case of the polytype $2 \mathrm{MC}$. All lines are present in both crystals at approximately the same frequencies. We suppose that these lines are due to internal vibrations of the network of $\mathrm{SbO}_{7}$ pyramids forming the slabs typical for all $\mathrm{Sb}_{5} \mathrm{O}_{7} \mathrm{I}$ polytypes $[2,3]$. This assumption is supported by the infrared transmission spectra and Raman spectra of $\mathrm{Sb}_{2} \mathrm{O}_{3}$, which show absorption lines at $340 \mathrm{~cm}^{-1}$ and $388 \mathrm{~cm}^{-1}$ and a very broad band around $492 \mathrm{~cm}^{-1}$ [15] and a Raman line at $419 \mathrm{~cm}^{-1}$ [16].

The absorption bands of $\mathrm{Sb}_{2} \mathrm{O}_{3}$ at $553 \mathrm{~cm}^{-1}, 597$ $\mathrm{cm}^{-1}, 695 \mathrm{~cm}^{-1}$ and $750 \mathrm{~cm}^{-1}$ have analogues in Reststrahlen bands found in the infrared reflection spectra of both $2 \mathrm{MC}$ - and 2MA-polytypes [12], but could not be detected in Raman scattering of $2 \mathrm{MA}$ $-\mathrm{Sb}_{5} \mathrm{O}_{7} \mathrm{I}$. Weak bands, however, are present at 600 $\mathrm{cm}^{-1}$ and at $750 \mathrm{~cm}^{-1}$ in the Raman spectra of $2 \mathrm{MC}$ $-\mathrm{Sb}_{5} \mathrm{O}_{7} \mathrm{I}$ [5].

Below the gap the $A^{\prime \prime}$ doublet at $182 \mathrm{~cm}^{-1}$ and 190 $\mathrm{cm}^{-1}$ corresponds to the $B_{\mathrm{g}}$ doublet of the $2 \mathrm{MC}$ modification at $183 \mathrm{~cm}^{-1}$ and $193 \mathrm{~cm}^{-1}$ [5]. $\mathrm{Sb}_{2} \mathrm{O}_{3}$ has a narrow absorption line at $181 \mathrm{~cm}^{-1}[17]$ and a Raman line at $180 \mathrm{~cm}^{-1}$ [16] indicating that these modes might also be caused by $\mathrm{Sb}-\mathrm{O}$ vibrations. Further coincidences are found for the strong 146 $\mathrm{cm}^{-1} A^{\prime}$ line and the $156 \mathrm{~cm}^{-1} A^{\prime}$ line which correspond to the $145 \mathrm{~cm}^{-1} A_{\mathrm{g}}$ mode and the $156 \mathrm{~cm}^{-1}$ $A_{g}$ mode of the $2 \mathrm{MC}$ modification, respectively. $\mathrm{Sb}_{2} \mathrm{O}_{3}$ shows a Raman line just at $145 \mathrm{~cm}^{-1}$, however these modes might also be due to $\mathrm{I}-\mathrm{Sb}$ vibrations because $\mathrm{SbI}_{3}$ has a broad absorption band around $150 \mathrm{~cm}^{-1}$ [17].

A list of frequencies of Raman scattering lines measured at room temperature of the polytype $2 \mathrm{MA}$ determined in this work and those of the polytype $2 \mathrm{MC}$ [5] are given in Table 1. In addition all Raman lines and infrared absorption bands of $\mathrm{Sb}_{2} \mathrm{O}_{3}$ known from the literature are listed [15-17].

\section{Symmetry Considerations}

\section{a) Factor Group Analysis}

In the ferroic phase we have observed a total of $18 A^{\prime}$ modes and $15 A^{\prime \prime}$ modes. The theoretically possible number of zone center phonons and their distribution among the irreducible representations of the point group of a crystal may be determined by a factor group analysis, which yields a decomposition of the total representation $\Gamma$ of the structure into irreducible representations. We will perform this analysis for both structural phases. The results for the prototypic phase will be used in the next section to determine the number of modes belonging to different symmetries in compatibility relations connecting the high and low temperature phases. The decomposition of the total representation $\Gamma_{f}$ of the ferroic phase is very easily performed by the following consideration. Each atom of this structure occupies a general 2-fold 
Table 2. Factor group analysis of $\mathrm{Sb}_{5} \mathrm{O}_{7} \mathrm{I}$ polytype $2 \mathrm{MA}$ in the prototypic phase

\begin{tabular}{|c|c|c|c|c|c|c|}
\hline \multicolumn{3}{|c|}{$C_{3 h}^{1}(P \overline{6})$ No. 174} & \multicolumn{4}{|c|}{ Number of irreducible representations } \\
\hline \multirow[t]{2}{*}{ Atom } & \multirow{2}{*}{$\begin{array}{l}\text { Site } \\
\text { sym- } \\
\text { metry }\end{array}$} & \multirow{2}{*}{$\begin{array}{l}\text { Wyck off } \\
\text { equi- } \\
\text { point }\end{array}$} & \multicolumn{2}{|c|}{ Raman active } & \multirow{2}{*}{$\begin{array}{l}\text { Raman } \\
\text { active, } \\
\text { infrared } \\
\text { active and } \\
\text { acoustic } \\
E^{\prime}\end{array}$} & \multirow{2}{*}{$\begin{array}{l}\text { infrared } \\
\text { active } \\
\text { and } \\
\text { acoustic } \\
A^{\prime \prime}\end{array}$} \\
\hline & & & $A^{\prime}$ & $E^{\prime \prime}$ & & \\
\hline $3 \mathrm{Sb}$ & $C_{s}$ & $3(k)$ & 2 & 1 & 2 & 1 \\
\hline $3 \mathrm{Sb}$ & $C_{s}$ & $3(j)$ & 2 & 1 & 2 & 1 \\
\hline $2 \mathrm{Sb}$ & $C_{3}$ & $2(i)$ & 1 & 1 & 1 & 1 \\
\hline $2 \mathrm{Sb}$ & $C_{3}$ & $2(h)$ & 1 & 1 & 1 & 1 \\
\hline $2 \times 60$ & $C_{1}$ & $6(l)$ & 6 & 6 & 6 & 6 \\
\hline $\mathrm{O}$ & $C_{3 h}^{1}$ & $1(e)$ & 0 & 0 & 1 & 1 \\
\hline $\mathrm{O}$ & $C_{3 h}$ & $1(d)$ & 0 & 0 & 1 & 1 \\
\hline & $C_{3}$ & $2(g)$ & 1 & 1 & 1 & 1 \\
\hline Total & & & 13 & 11 & 15 & 13 \\
\hline
\end{tabular}

Wyckoff equivalent point (e) with site symmetry $C_{1}$ [3]. Therefore the 6 vibrational degrees of freedom of a group of two identical atoms on equivalent positions are distributed equally among the two irreducible representations $A^{\prime}$ and $A^{\prime \prime}$ of $C_{2}$. Thus we find after subtracting the translation of the crystal as a whole:

$\Gamma_{f}=76 A^{\prime}+77 A^{\prime \prime}$

(Raman active and infrared active).

This shows that we have observed at room temperature, as in the case of the polytype $2 \mathrm{MC}$, for both symmetry species only a fraction of all possible Raman active phonons.

The total representation $\Gamma_{p}$ of the prototypic phase may be reduced by application of the tables of Adams and Newton [18]. For each atom in the high temperature hexagonal phase the site symmetry, the Wyckoff equipoint and the contribution of its translational degrees of freedom to the irreducible representations of the point group $C_{3 h}$ are listed in Table 2. From this the decomposition of $\Gamma_{p}$ is obtained:

$$
\begin{aligned}
\Gamma_{p}= & 13 A^{\prime}+11 E^{\prime \prime} & & \text { (Raman active) } \\
& +14 E^{\prime} & & \text { (Raman and infrared active) } \\
& +12 A^{\prime \prime} & & \text { (infrared active) }
\end{aligned}
$$

where the acoustic modes have again been subtracted.

\section{b) Compatibility Relations}

Due to the doubling of the unit cell and the reduction of the point symmetry connected with the appearance
Table 3. Compatibility relations of the normal modes along the $\Sigma$ axis of the prototypic $C_{3 h}^{1}$-phase with respect to the recuction of the symmetry to $C_{s}^{2}$ which is connected with a folding of the Brillouin zone normal to the $\Sigma$-axis. The compatibility of $\Gamma$-point and $M$-point representations of $C_{3 h}^{1}$ with $\Gamma$-point representations of $C_{s}^{2}$ is given at the right and left side. The numbering of the $\Sigma$ and $M$-species is after Zak [24]

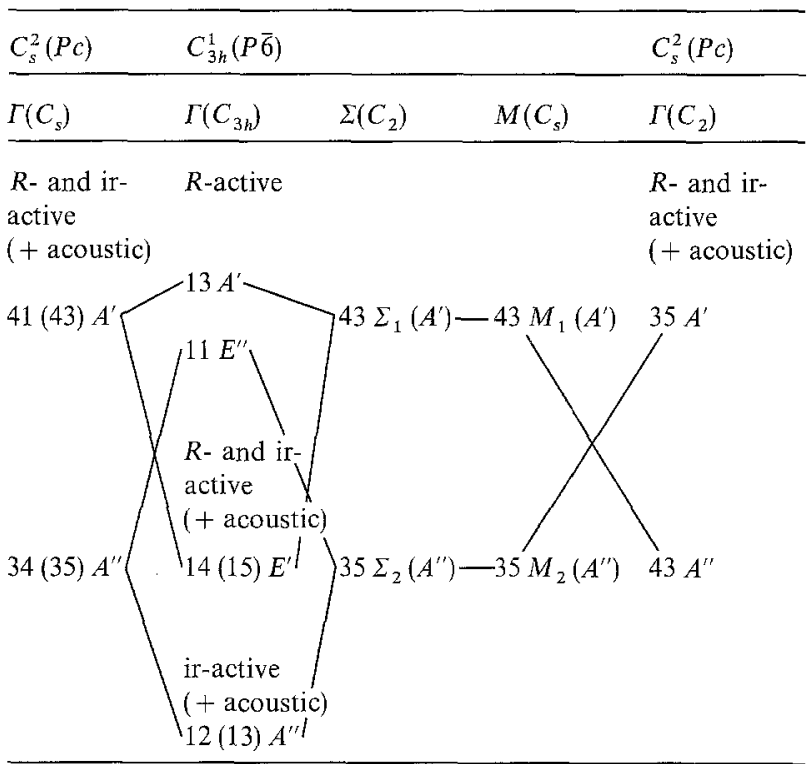

of the spontaneous polarization and deformation, phonons which are inactive in the prototypic phase may be Raman active below $T_{c}$. In particular a potential soft mode, which is in all cases Raman active in a ferroic phase, must be a zone boundary phonon at the $M$-point of the high temperature structure. In order to determine the number and the symmetries of the phonons in the ferroic phase as they come out from zone center and $M$-point modes of the hexagonal phase the compatibility relations along the $\Sigma$-axis of the hexagonal Brillouin zone have been analyzed and the reduction of the zone center and zone boundary species in terms of the representations of the point group $C_{s}$ of the ferroic phase has been determined. The results are given in Table 3. The factor groups of the wavevector along the $\Sigma$-axis and at the $M$-point are isomorphic to the point group $C_{s}$ and the character tables are identical to that of $C_{s}$, because the space group $C_{3 h}^{1}$ is symmorphic. Therefore we have added the corresponding point group notations in brackets behind the factor group species of the $\Sigma$-axis and the $M$-point. Table 3 may be obtained by simply applying point group compatibility relations. Proceeding from the $M$ point of $C_{3 h}^{1}$ to the $\Gamma$ point of $C_{s}^{2}$, the species $A^{\prime}$ and $A^{\prime \prime}$ are interchanged though both factor groups are isomorphic to $C_{s}$. As in the case of $2 \mathrm{MC}-\mathrm{Sb}_{5} \mathrm{O}_{7} \mathrm{I}$ [5] this is due to the fact that $\tau=\mathbf{a}_{2}=\mathbf{c} / 2$ is a primitive translation of $C_{3 h}^{1}$, but a fractional translation of $C_{s}^{2}$. 


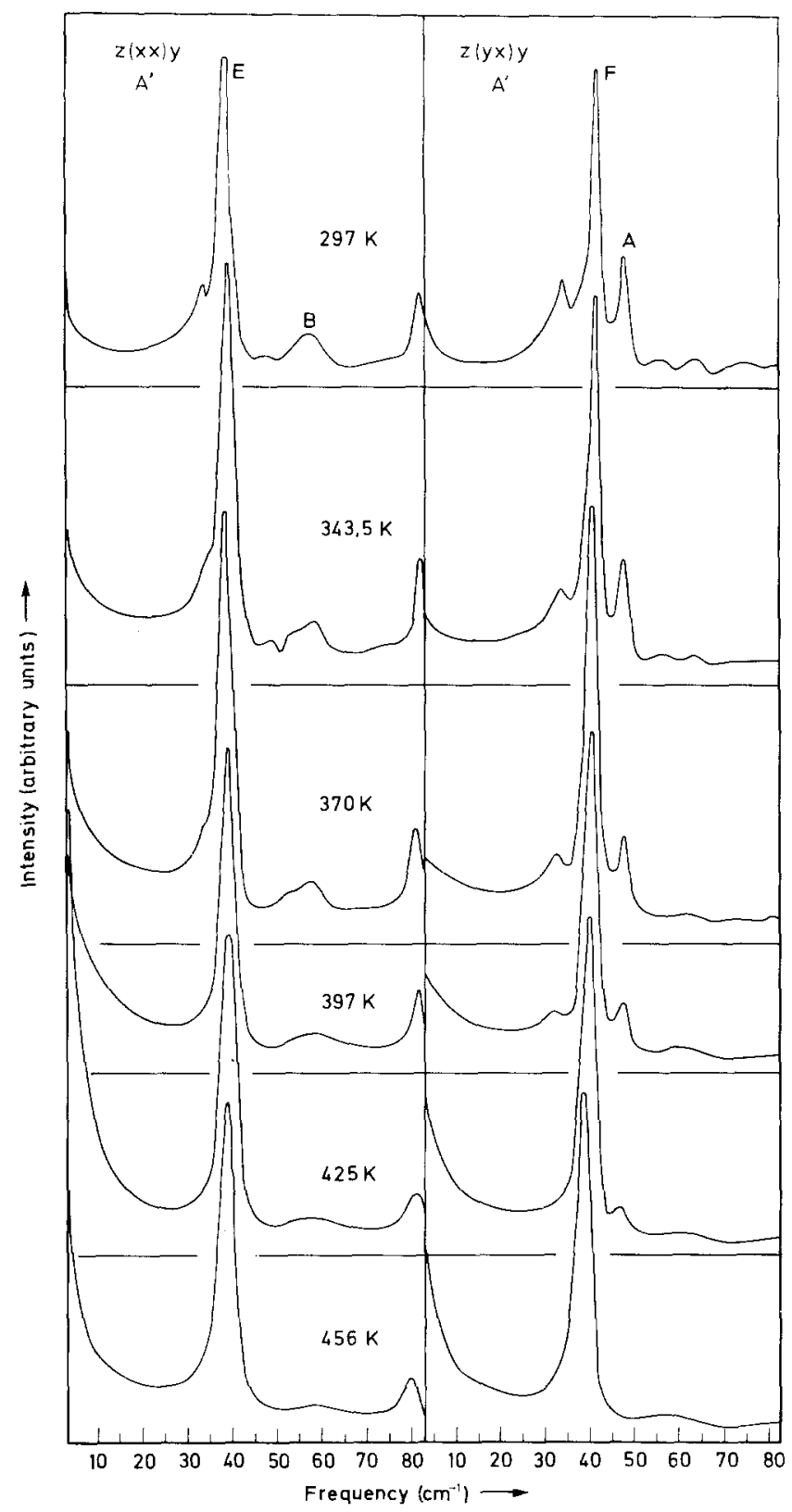

Figs. 3 and 4. Low frequency Raman spectra of $\mathrm{Sb}_{5} \mathrm{O}_{7} \mathrm{I}$ polytype $2 \mathrm{MA}$ at various temperatures below and above $T_{\mathrm{c}}=438 \mathrm{~K}$

Table 3 shows that optical phonon branches yield $A^{\prime}$ and $A^{\prime \prime}$ modes in the ferroic phase always in pairs. Because optical branches, particularly at higher frequencies, are usually flat, this might explain the observation that close to the frequencies of one species weak lines of the other symmetry are always found. Further, we see that $A^{\prime}$ modes losing Raman activity above $T_{c}$ always originate from zone boundary phonons, whereas $A^{\prime \prime}$ modes activated by the phase transition may come out both from zone center and zone boundary phonons of the prototypic phase. The order parameter of the phase-transition, which

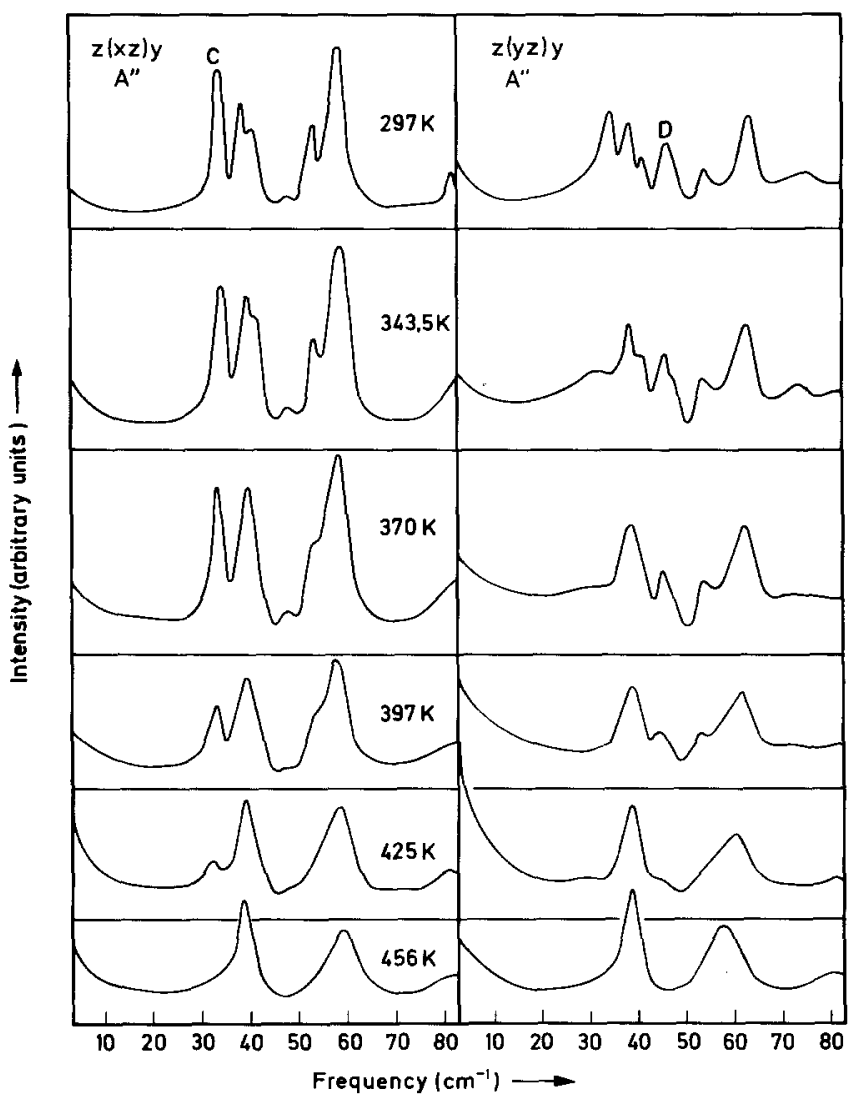

describes the structural distortion of the crystal below $T_{c}$, must belong to the $M_{2}\left(A^{\prime \prime}\right)$ representation at the $M$-point.

\section{Temperature Dependence of the Raman Spectra}

The temperature dependence of the Raman spectra have been determined from room temperature to well above $T_{c}=438 \mathrm{~K}$. The results are shown in Fig. 3 and Fig. 4 for the scattering configuration $z\left(x_{i} x_{j}\right) y$. Only the spectral range between 3 and $83 \mathrm{~cm}^{-1}$ is displayed, which is sufficient to discuss typical changes of the scattering intensities. First of all we note that in the $A^{\prime}$ spectra, where a totally symmetric soft mode should appear below $T_{c}$, no indication of soft phonons can be found. This is in contrast to the polytype $2 \mathrm{MC}$ where a heavily damped soft mode has been observed $[5,6]$. But as in the case of $2 \mathrm{MC}$ $-\mathrm{Sb}_{5} \mathrm{O}_{7} \mathrm{I}$, the phase transition is accompanied by a strongly temperature dependent quasielastic scattering, whose intensity peaks around $T_{c}$ and decreases above $T_{c}$. This scattering is definitely present above the phase transition in the high temperature phase. Therefore it cannot solely originate from elastic scatering by macroscopic domain boundaries which might be formed in the ferroic phase close to $T_{c}$. 


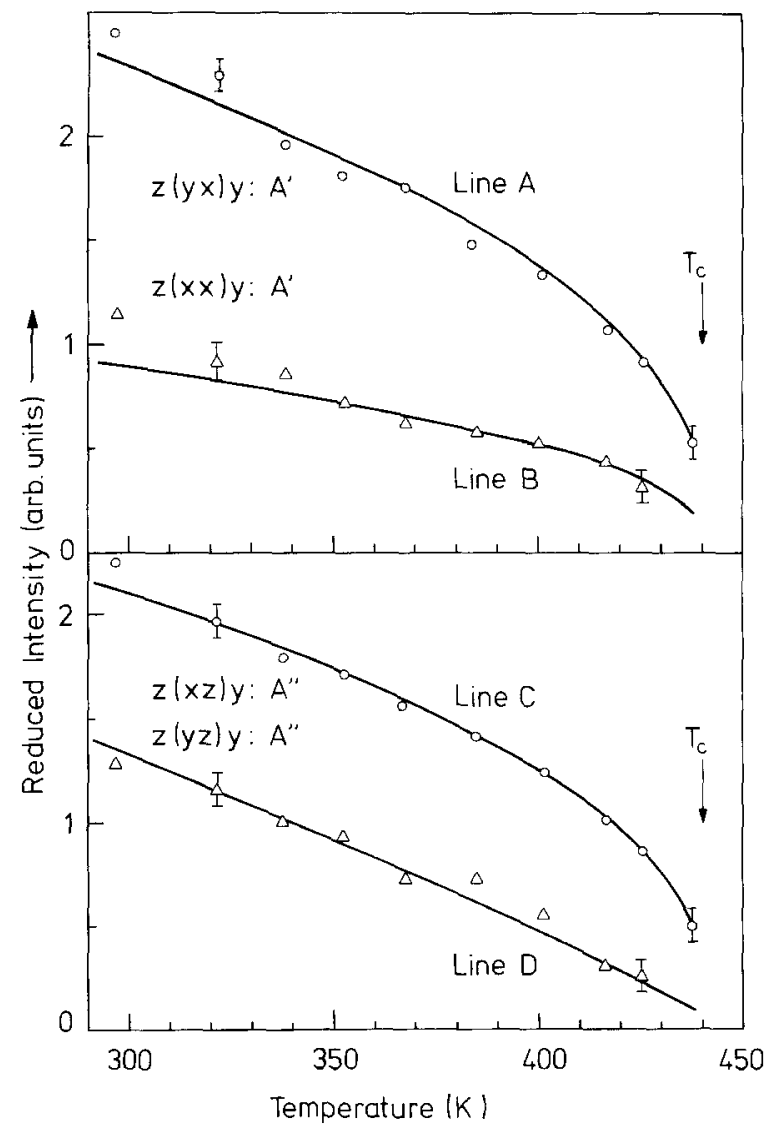

Fig. 5. Temperature dependence of the reduced intensity $I_{\text {red }}$ $=I /(n(\omega)+1)$ for the lines $A, B, C$ and $D$ (Figs. 2-4). The circles and triangles are experimentally determined values, the full lines are best-fit curves according to Eqs. (4) and (5)

Below $T_{c}$ the observed quasielastic scattering can also be due to not resolved Brillouin scattering by transverse acoustic phonons, whose frequency will be approaching $T_{c}$ by the quadratic coupling of the zone boundary order parameter to elastic deformations. Above $T_{c}$ such a coupling does not exist. Therefore we assume that the quasielastic scattering belongs to some extent to a central line, as has been observed in conjunction with structural phase transitions in many other crystals [19]. The presence of a central line in $2 \mathrm{MC}-\mathrm{Sb}_{5} \mathrm{O}_{7} \mathrm{I}$ has already been proved by neutron scattering [12]. Several theoretical explanations for the central line have been proposed based on very different physical mechanisms. Recently Halperin and Varma [19] have shown that a certain type of defects may produce a central peak with the extremely narrow width usually observed. Obviously Raman measurements cannot reveal the cause of the central peak in $2 \mathrm{MA}-\mathrm{Sb}_{5} \mathrm{O}_{7} \mathrm{I}$ and the reason why it is not coupled with an observable soft phonon. It should be noted, however, that the phase transition temperature in all $\mathrm{Sb}_{5} \mathrm{O}_{7} \mathrm{I}$ polytypes is very sensitive to substitution of antimony by arsenic. Incorporation of a few percent of As lowers $T_{c}$ by 50 to $100 \mathrm{~K}$ [3]. Investigations of the effect of these impurities on the central line scattering are under progress.

In the spectra of Fig. 3 and Fig. 4 six lines are indicated by the letters $A$ to $F$. The intensities of the four lines $A$ to $D$ decrease with rising temperature and vanish at $T_{c}$. In Fig. 5 the reduced peak intensities $I_{\text {red }}=I /(n(\omega)+1)$ of these lines as a function of temperature are shown, where $I$ is the intensity and $n(\omega)$ is the Bose population factor. The lines $A$ to $D$ result either from Raman inactive zone center or zone boundary phonons of the hexagonal structure which are activated below $T_{c}$ by the appearance of the spontaneous polarization and strain or by the folding of the Brillouin zone. The compatibility relations (Table 2) show, that the $A^{\prime}$-modes $A$ and $B$ must belong to the $M_{2}\left(A^{\prime \prime}\right)$ representation of the $M$ point of the hexagonal structure whereas the $A^{\prime \prime}$ phonons $C$ and $D$ may be $A^{\prime \prime}$-modes of the zone center or $M_{1}\left(A^{\prime}\right)$-modes of the zone boundary $M$ point of the prototypic phase. The relation between the intensity of Raman lines activated by the phase transition and the order parameter may be deduced in the same way as it was done for $2 \mathrm{MC}-\mathrm{Sb}_{5} \mathrm{O}_{7} \mathrm{I}$ [6].

The free energy $F$ of $2 \mathrm{MA}-\mathrm{Sb}_{5} \mathrm{O}_{7} \mathrm{I}$ has the same form as that of the 2MC-polytype except that an additional coupling term

$$
\begin{aligned}
& F_{p}=e\left[P_{x}\left(2 Q_{3}^{2}-Q_{2}^{2}-Q_{1}^{2}\right)+P_{y} 3^{\frac{1}{2}}\left(Q_{2}^{2}-Q_{1}^{2}\right)\right] \\
& +f\left[P_{x}\left(u_{x x}-u_{y y}\right)+P_{y} 2 u_{x y}\right]
\end{aligned}
$$

is allowed by symmetry and yields the ferroelectric properties of the crystal. In this expression $e$ and $f$ are expansion coefficients, $\left(P_{x}, P_{y}\right)$ and $\left(u_{x x}-u_{y y}, 2 u_{x y}\right)$ are components of the electric polarization $\mathbf{P}$ and the elastic deformation $\mathbf{u}$, respectively, which are basis functions of the $E^{\prime}$-representation of $C_{3 h}$ and $\mathbf{Q}$ $=\left(Q_{1}, Q_{2}, Q_{3}\right)$ is the three-component order parameter, where each $Q_{\mu}$ is associated with one wavevector of the threefold star of the $M$-point. After elimination of $\mathbf{P}$ and the strain $\mathbf{u}$ the same expression for $F$ as in the case of $2 \mathrm{MC}-\mathrm{Sb}_{5} \mathrm{O}_{7} \mathrm{I}$ is found [6]:

$$
\begin{aligned}
& F(\mathbf{Q})=\frac{1}{2} \alpha \Sigma Q_{\mu}^{2}+\frac{1}{4} \beta_{1} \Sigma Q_{\mu}^{4}+\frac{1}{2} \beta_{2}\left(Q_{2}^{2} Q_{3}^{2}\right. \\
& \left.+Q_{1}^{2} Q_{3}^{2}+Q_{1}^{2} Q_{2}^{2}\right)+f_{6}(\mathbf{Q})
\end{aligned}
$$

where $f_{6}(\mathbf{Q})$ contains sixth order invariants of $\mathbf{Q}$. As usual we assume that $\alpha$ varies with temperature as $\alpha \propto T-T_{0}$ and the coefficients $\beta_{1}$ and $\beta_{2}$ are independent of $T$ close to the phase transition. By minimizing $F$ with respect to $Q_{\mu}$ the solutions $\mathbf{Q}^{s}=$ $( \pm \eta, 0,0),(0, \pm \eta, 0),(0,0, \pm \eta)$ are found to be stable below the transition temperature if the coefficients in 
Eq. (5) fullfill certain conditions discussed in Ref. 6 . Each solution gives one of the three domains of the ferroic phase.

The spontaneously appearing elastic deformation $\mathbf{u}^{s}$ and electric polarization $\mathbf{P}^{s}$ are proportional to $\eta^{2}$ :

$u_{x x}^{s}-u_{y y}^{s}, 2 u_{x y}^{s} \propto \eta^{2} ; \quad P_{x}^{s}, P_{y}^{s} \propto \eta^{2}$

as is usually the case for antiferrodistortive phase transitions.

In passing we note that the free energy Eq. (5) has just the same form as that of perovskite structure crystals undergoing antiferrodistortive structural changes like $\mathrm{SrTiO}_{3}, \mathrm{LaAlO}_{3}$ etc. [20]. However in contrast to the perovskite structure the solution $\mathbf{Q}^{s}=3^{-\frac{1}{2}} \eta( \pm 1$, $\pm 1, \pm 1$ ), which yields the trigonal distortion in $\mathrm{LaAlO}_{3}$ and other aluminates $[21,20]$ and which is essential for the three-state Potts transition [22] in stressed $\mathrm{SrTiO}_{3}$ [23], does not occur in 2MA- or $2 \mathrm{MC}-\mathrm{Sb}_{5} \mathrm{O}_{7} \mathrm{I}$. This solution retains the hexagonal symmetry and does not induce a spontaneous strain or polarization. Further, we want to point out that $2 \mathrm{MA}-\mathrm{Sb}_{5} \mathrm{O}_{7} \mathrm{I}$ is not a zero field realization of the three-state Potts model, though the three-domain structure of $\mathbf{P}^{s}$ in the ferroic phase strongly resembles the three-state Potts symmetry [25]: $\mathbf{P}^{s}$ has three equilibrium directions symmetrically placed in the hexagonal plane of the crystal and the free energy expanded in powers of $\mathbf{P}$ contains a third order term of the form $P_{x}\left(P_{x}^{2}-3 P_{y}^{2}\right)$, which is responsible for the discontinuous character of the three-state Potts transition $[25,23]$. However in $2 \mathrm{MA}-\mathrm{Sb}_{5} \mathrm{O}_{7} \mathrm{I} \mathbf{P}$ is not the order parameter; $P_{x}$ and $P_{y}$ are proportional to the square of the order parameter as given in Eq. (6). Therefore the above expression is actually a sixth order term of the order parameter.

By expanding the Raman polarizability $\mathbf{R}$ in terms of $\mathbf{Q}, \mathbf{u}^{s}$ and $\mathbf{P}^{s}$ it is found that $\mathbf{R}$ is proportional to $\eta$ for zone boundary phonons and proportional to $\mathbf{u}^{s}$ and $\mathbf{P}^{s}$ for zone center modes.

Therefore the reduced intensity turns out to be

$I_{\text {red }}=a_{2} \eta^{2} \quad$ for zone boundary modes,

$I_{\text {red }}=a_{4} \eta^{4} \quad$ for zone center modes,

where the coefficients $a_{2}$ and $a_{4}$ are assumed to be independent of temperature. The experimentally determined intensities of the lines $A, B, C$ and $D$ vanish discontinuously at $T_{c}$ as it is most clearly observed from line $A$ and line $C$ (Fig. 5). This shows that the phase transition of $2 \mathrm{MA}-\mathrm{Sb}_{5} \mathrm{O}_{7} \mathrm{I}$ is of first order with a small discontinuity of the order parameter of the transition temperature. The same result was found for $2 \mathrm{MC}-\mathrm{Sb}_{5} \mathrm{O}_{7} \mathrm{I}$ [6]. Weakly discontinuous phase transitions may be well described by the classical Landau theory. In this case the order parameter varies with temperature as

$\eta^{2}(T)=\eta^{2}\left(T_{1}\right)\left\{1+\left[\left(T_{1}-T\right) /\left(T_{1}-T_{0}\right)\right]^{\frac{1}{2}}\right\}$

where $T_{1}$ and $T_{0}$ are the boundaries of stability of the low temperature and high temperature phases, respectively, and $T_{1}-T_{c}=\left(T_{c}-T_{0}\right) / 3 . T_{c}$ is the temperature where both phases have the same free energy.

We have fitted the measured intensities of the lines $A$, $B$ and $C$ according to Eq. (7a) and that of line $D$ according to Eq. (7b) by varying $\eta\left(T_{1}\right)$ and $\Delta T=T_{1}$ $-T_{0}$ (Eq. 8). The results are displayed in Fig. 5 by the full lines and agree rather well with the experimental points. As expected from the compatibility relations of Table 3, the $A^{\prime}$ lines $A$ and $B$ behave like $\eta^{2}$ and thus result from $M_{2}\left(A^{\prime \prime}\right)$ zone boundary modes of the hexagonal phase. The $A^{\prime \prime}$ line $C$ behaves also like $\eta^{2}$ showing that this mode is also activated at the zone boundary of the high temperature phase but belongs to the $M_{1}\left(A^{\prime}\right)$ species above $T_{c}$. The intensity of the $A^{\prime \prime}$ line $D$ varies like $\eta^{4}$. Therefore this line must belong to a $\Gamma$-point phonon belonging to the $A^{\prime \prime}$ representation above $T_{c}$, the only Raman inactive irreducible zone center representation of the prototypic structure. The temperature interval $\Delta T=T_{1}$ $-T_{0}$ is a measure of the discontinuity of the phase transition and gives the extent of the metastability region where both phases may exist. The best fit for all four curves was obtained by using $\Delta T=4 \mathrm{~K}$.

The frequencies of the $A^{\prime}$ lines $E$ and $F$ at $297 \mathrm{~K}$ are $38 \mathrm{~cm}^{-1}$ and $41.5 \mathrm{~cm}^{-1}$, respectively. The frequency difference decreases continuously approaching the phase transition and both lines coincide above $T_{c}$ at $38.5 \mathrm{~cm}^{-1}$. This shows that these lines result from doubly degenerate $E^{\prime}$ modes at the $\Gamma$-point of the hexagonal structure. The frequency splitting in the ferroic phase is proportional to the square of the order parameter.

The authors are indebted to Dipl. Phys. M. Schuhmacher and Dr. V. Krämer for providing the samples and helpful comments on the properties and the structure of $\mathrm{Sb}_{5} \mathrm{O}_{7} \mathrm{I}$ polytypes. Stimulating discussions with Prof. U. Krey and Dr. A. Baratoff are gratefully acknowledged.

\section{References}

1. Krämer, V., Nitsche, R., Schuhmacher, M.: J. Cryst. Growth 24/25, 179 (1974)

2. Krämer, V.: Acta Cryst. B31, 234 (1975)

3. Nitsche, R., Krämer, V., Schuhmacher, M., Bussmann, A.: J. Cryst. Growth 42, 549 (1977)

4. Aizu, K.: J. Phys. Soc. Japan 27, 387 (1969)

5. Prett1, W., Rieder, K.H., Nitsche, R.: Z. Physik B 22, 49 (1975)

6. Prettl, W., Rieder, K.H.: Phys. Rev. B 14, 2171 (1976) 
7. Prettl, W., Rieder, K.H., Nitsche, R.: Proc. of the 5th Int. Conf. on Raman Spectroscopy, Freiburg 1976, p. 618. The discussion in this report based upon the space groups $C_{2 v}^{10}$ and $D_{3 h}^{4}$ for the ferroic and prototypic structure respectively. Recent crystallographic investigations by Krämer (unpublished, see also [3]) have proved the space groups quoted in the present paper to be correct

8. Steinbrener, S.: private communication

9. Levanyuk, A.P., Sannikov, D.G.: Fiz. Tverd Tela 12, 2997 (1970)

10. Dvorák, V.: phys. stat. sol. b 45,147 (1971)

11. Aizu, K.: J. Phys. Soc. Japan 32, 1287 (1972)

12. Prettl, W.: to be published

13. Loudon, R.: Adv. Phys. 13, 423 (1967)

14. Shapiro, S.M., Axe, J.D.: Phys. Rev. B 6, 2420 (1972)

15. Miller, F.H., Carlson, G.L., Bentley, F.F., Jones, W.H.: Spectrachimica Acta 16, 135 (1960)

16. Chevemisinov, V.P.: Proc. P.N. Lebedev Physics Institute 25 , 129 (1965)

17. Nyquist, R.A., Kagel, R.O.: Infrared Spectra of Organic Compounds. New York and London: Academic Press 1971

18. Adams, D.M., Newton, D.C.: Tables for Factor Group and Point Group Analysis. Cryodon (Great Britain): BeckmanRIIC Limited 1970

19. Halperin, B.I., Varma, C.M.: Phys. Rev. B 14, 4040 (1976)

20. Slonczewski, J.C., Thomas, H.: Phys. Rev. B 1, 3599 (1970)
21. Derighetti, B., Drumheller, J.E., Laves, F., Müller, K.A., Waldner, F.: Acta Cryst. 18, 557 (1965)

22. Potts, R.B.: Proc. Cambridge Philos. Soc. 48, 106 (1952)

23. Aharony, A., Müller, K.A., Berlinger, W.: Phys. Rev. Lett. 38, 33 (1977)

24. The Irreducible Representations of Space Groups, ed. J. Zak. New York: Benjamin 1971

25. Golner, R.G.: Phys. Rev. B 8, 3419 (1973)

\section{W. Prettl}

Institut für Angewandte Physik

der Universität Regensburg

Universitätsstr. 31

D-8400 Regensburg

Federal Republic of Germany

K.H. Rieder

IBM Zurich Research Laboratory

CH-8803 Rüschlikon

Switzerland

R. Nitsche

Kristallographisches Institut

der Universität

Hebelstr. 25

D-7800 Freiburg

Federal Republic of Germany 